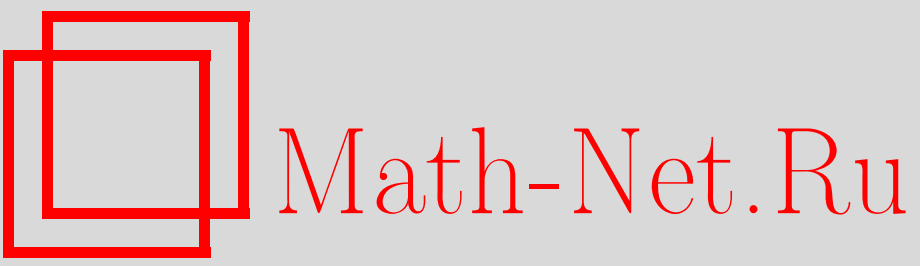

В. М. Бухштабер, А. Ю. Лазарев, Дискретное преобразование Гельфанда и двойственное к нему, УМН, 2004, том 59, выпуск 1, 183-184

DOI: https://doi.org/10.4213/rm710

Использование Общероссийского математического портала Math-Net.Ru подразумевает, что вы прочитали и согласны с пользовательским соглашением

http://www.mathnet.ru/rus/agreement

Параметры загрузки:

IP : 54.224 .60 .19

26 апреля 2023 г., 05:03:21 


\title{
ДИСКРЕТНОЕ ПРЕОБРАЗОВАНИЕ ГЕЛЬФАНДА И ДВОЙСТВЕННОЕ К НЕМУ
}

\author{
В. М. БУХШТАБЕР, А. Ю. ЛАЗАРЕВ
}

Пусть $L$ - коммутативное кольцо без делителей нуля и $K$ - бесконечное подкольцо в $L$. Рассмотрим некоторую $K$-алгебру $A$, являющуюся свободным $K$-модулем, и множество ее $K$-точек $A(K)$, т.е. множество всех гомоморфизмов $K$-алгебр $A \rightarrow K$. Пусть $L[A(K)]$ - свободный $L$-модуль, натянутый на множество $A(K)$, и $\mathrm{Map}(A(K), L)$ - кольцо всех функций $A(K) \rightarrow L$.

Дискретным преобразованием Гельфанда будем называть отображение

$$
e v_{L / K}: A \otimes L \rightarrow \operatorname{Map}(A(K), L), \quad e v(a \otimes l)(x)=l x(a) .
$$

Кольо $\operatorname{Map}(A(K), L)$ имеет каноническую структуру топологического $L$-модуля с системой окрестностей нуля $U_{X}$, где $X$ пробегает все конечные подмножества в $A(K)$, а $U_{X}$ - идеал функций, обращающихся в нуль на $X$. Используя непрерьвные $L$-гомоморфизмы, получаем двойственное преобразование

$$
e v_{L / K}^{*}: L[A(K)] \rightarrow(A \otimes L)^{*}:=\operatorname{Hom}_{K}(A, L),
$$

принимающее значения в топологическом $L$-модуле с системой окрестностей нуля $U_{W}$, где $W$ пробегает конечномерные $K$-подмодули $W$ в $A$, а $U_{W}$ состоит из всех $K$-гомоморфизмов $A \rightarrow L$, обращающихся в нуль на $W$.

УТВЕРЖДЕНИЕ 1. Гомоморфизм $е v_{L / K}^{*}$ является мономорфизмом.

Доказательство опирается на так назьваемый трюк Артина, при помощи которого показывается линейная независимость характеров, см. [1]. Этот трюк применим в нашем более общем случае, так как $e v_{L / K}^{*}$ является гомоморфизмом $A$-модулей. Имеют место очевидные вложения симметрических степеней $i_{n}: \operatorname{Sym}^{n}(A(K)) \subset L[A(K)], n=1,2, \ldots$. Описание композиции $e v_{L}^{*} / K i_{n}$ в терминах $n$-гомоморфизмов, обобщающих высшие характеры $\Phi$ робениуса, дано в [2].

ОПРЕДЕЛЕниЕ 1. Пусть $\widehat{L}$ - поле частных кольца $L$. Кольцом числовых функций $\operatorname{Num}_{L / K}(A)$ назьвается подкольцо в $A \otimes \widehat{L}$, состоящее из всех элементов, которые при отображении $e v \widehat{L} / K$ переходят в функции $A(K) \rightarrow L$.

$L$-модулем числовых функционалов $\mathcal{N} \mathrm{um}_{L / K}(A)$ назьвается $L$-подмодуль в $\widehat{L}[A(K)]$, состоящий из всех элементов, которые при отображении $e v \stackrel{\leftarrow}{*} / K$ переходят в $K$-гомоморфизмы $A \rightarrow L$.

Положим $\operatorname{Num}_{L / K}(K[x])=\operatorname{Num}_{L / K}$ и $\mathcal{N u m}_{L / K}(K[x])=\mathcal{N u m}_{L / K}$.

ПримеР 1. Кольцо $\mathrm{Num}_{\mathbb{Z} / \mathbb{Z}}$ состоит из полиномов $p(x)$ с рациональными коэффицциентами, которые принимают целые значения при целых $x$. Это кольцо аддитивно порождено классическими числовыми полиномами $P_{k}(x)=x(x-1) \cdots(x-k+1) / k !, k=0,1,2, \ldots$.

Колшц $\mathrm{Num}_{\mathbb{Z} / \mathbb{Z}}$ нашло важные приложения в алгебраической топологии, см. [3]-[5]. В общем случае задача описания колец $\operatorname{Num}_{L / K}$ не решена. В то же время для $\mathcal{N} \mathrm{um}_{L / K}$ имеет место следующий общий результат.

Пусть $\omega=\left(k_{1}, \ldots, k_{n}\right)$ - набор из $n$ попарно различных элементов кольца $K$. Рассмотрим матрицу Вандермонда $V_{\omega}=\left(k_{i}^{j}, 1 \leqslant i \leqslant n, 0 \leqslant j \leqslant n-1\right)$ и обозначим через $V_{l, \omega}$ матрицу, полученную из $V_{\omega}$ заменой $l$-й строки строкой $\left(\left[k_{1}\right], \ldots,\left[k_{n}\right]\right)$, где $\left[k_{i}\right]-$ образующий кольца $L[K]$, соответствующий элементу $k_{i}$. Положим $e_{l, \omega}=\frac{\operatorname{det} V_{l, \omega}}{\operatorname{det} V_{\omega}} \in \widehat{L}[K]$.

Tеорема 1. $e_{l, \omega} \in \mathcal{N u m}_{L / K}$, и любой әлемент из $\mathcal{N u m}_{L / K} \subset \widehat{L}[K]$ вида $\sum \alpha_{i}\left[k_{i}\right]$ принадлежит свободному $L$-модулю, порожденному әлементами $e_{l, \omega}$, где $1 \leqslant l \leqslant n$.

Теорема 2. Следующие условия әквивалентны: (1) $\mathcal{N u m}_{L / K}=L[K] ;$ (2) образ отображения еv ${ }_{L / K}^{*}: L[x] \rightarrow \operatorname{Map}(K, L)$ всюду плотен; (3) кольцо L содержит поле частных $\widehat{K}$ кольца $K$.

Работа выполнена при поддержке EPSRC (грант GR/R84276/01). 
ОПРедЕЛЕНИЕ 2. Пусть $Q$ некоторое подкольо коммутативного кольца $R$ без делителей нуля. Скажем, что $R$ является DS-расширением кольца $Q$, если существует бесконечная последовательность элементов $q_{i} \in Q, i=1,2, \ldots$, такая, что $\left(q_{i}-q_{j}\right)$ обратимы в $R$ для всех $i \neq j$.

ПримеР 2. Любое DS-расширение кольца $\mathbb{Z}$ содержит поле рациональных чисел $\mathbb{Q}$.

ПримеР 3. Кольцо Новикова $\mathbb{Z}((q))$ формальных рядов Лорана с цельми коэффицциентами является DS-расширением кольца полиномов $\mathbb{Z}[q]$.

Теорема 3. Рассмотрим следующие условия: (1) L является DS-расширением кольча $K$; (2) образ отображения еv ${ }_{L / K}: L[K] \rightarrow L[x]^{*}$ всюду плотен; (3) $\operatorname{Num}_{L / K}=L[x]$; (4) для любого $a \in L$ образ композичии $K \hookrightarrow L \rightarrow L /(a)$ бесконечен. Тогда $(1) \Rightarrow(2) \Rightarrow$ $(3) \Rightarrow(4)$. Более того, если $L$ - факториальное кольцо, то (4) $\Rightarrow(3)$, и если $L$ - кольцо дискретного нормирования, то (4) $\Rightarrow(1)$.

Приложения к алгебрам когомологических операций. Пусть $N(R)$ - группа формальных степенных рядов $\left\{x(t)=t+\sum x_{k} t^{k+1}, x_{k} \in R\right\}$ относителшно подстановки ряда в ряд. Эта группа играет важную роль, в том числе в алгебраической топологии (см. [6]-[8]) и теории групп (см. [9]). Группа $N(R)$ является группой $R$-точек градуированной алгебры Хопфа $N=\mathbb{Z}\left[x_{1}, x_{2}, \ldots\right], \operatorname{deg} x_{i}=2 i$, с диагональю $\Delta x_{k}=\sum x_{i} \otimes\left[x(t)^{i+1}\right]_{k+1}$, где $[y(t)]_{j}-$ коэффициент при $t^{j}$ у ряда $y(t)$. Известная в теории комплексных кобордизмов алгебра Ландвебера-Новикова $S$ является градуированной алгеброй Хопфа, градуированно двойственной над $\mathbb{Z}$ алгебре $N$. Исползуя все гомоморфизмы, но уже без учета градуировки, получаем топологические неградуированные алгебры Хопфа $\operatorname{Hom}(N, \mathbb{Z})=\widetilde{S}$ и $\operatorname{Hom}(N, R) \cong \widetilde{S} \widehat{\otimes} R$.

СледствиЕ 1. Пусть $R$ - DS-расширение кольца $Q$. Тогда топологическая $R$-алгебра Хопфа $\widetilde{S} \widehat{\otimes} R$ содержит всюду плотную $R$-подалгебру Хопфа, изоморфную $R[N(Q)]$.

Пусть теперь $R$ - некоторая алгебра над полем вычетов $\mathbb{F}_{p}$. Обозначим через $P(R)$ группу формальных рядов вида $\sum x_{i} t^{p^{i}}$, где $x_{i} \in R$ и $x_{0}=1$. Группа $P(R)$ является группой $R$-точек градуированной алгебры Хопфо $P=\mathbb{F}_{p}\left[x_{1}, x_{2}, \ldots\right], \operatorname{deg} x_{i}=2\left(p^{i}-1\right)$, если $p>2$, и $\operatorname{deg} x_{i}=$ $2^{i}-1$ при $p=2$, с диагоналю $\Delta x_{k}=x_{k} \otimes 1+1 \otimes x_{k}+\sum x_{i} \otimes x_{k-i}^{p^{i}}$. Градуированная алгебра Хопфа, градуированно двойственная над $\mathbb{F} p$ алгебре $P$, изоморфна алгебре квадратов Стинрода $\mathscr{A}_{2}$ при $p=2$ и алгебре приведенных степеней Стинрода $\mathscr{A}_{p}$ при $p>2$. Как и выше, введем топологические неградуированные алгебры Хопфо $\operatorname{Hom}(P, \mathbb{F} p)=\widetilde{\mathscr{A}}$ и $\operatorname{Hom}(P, R) \cong \widetilde{A} \widehat{\otimes} R$.

СледствиЕ 2. Пусть $R$ - некоторое $D S$-расширение $\mathbb{F}_{p}$-алгебры $Q$. Тогда топологическая $R$-алгебра Хопфа $\widetilde{\mathscr{A}} \widehat{\otimes} R$ содержит всюду плотную $R$-подалгебру Хопфа, изоморфную $R[P(Q)]$.

\section{СПИСОК ЛИТЕРАТУРЫ}

[1] S. Lang. Algebra. Reading, MA: Addison-Wesley, 1984. [2] V.M. Buchstaber, E. G. Rees // Selecta Math. (N.S.). 2002. V. 8. № 4. P. 523-535. [3] A. Baker, F. Clarke, N. Ray, L. Schwartz // Trans. Amer. Math. Soc. 1989. V. 316. № 2. P. 385-432. [4] T. Ekedahl // Homology Homotopy Appl. 2002. V. 4. № 2, part 1. P. 191-218 (electronic). [5] J. Hubbuck // J. London Math. Soc. (2). 1997. V. 55. № 1. P. 65-75. [6] В. М. Бухштабер, А. В. Шокуров // Функц. анализ и его прил. 1978. Т. 12. № 3. C. 1-11. [7] V. Buchstaber // Amer. Math. Soc. Transl. Ser. 2. 1995. V. 170. P. 9-31. [8] D. Ravenel. Complex Cobordism and Stable Homotopy Groups of Spheres. Orlando, FL: Academic Press, 1986. [9] R. Camina. The Nottingham group. New horizons in pro- $p$ groups // Progr. Math. 2000. V. 184. P. 205-221.

Математический институт им. В. А. Стеклова РАН;

Бристольский университет

Принято редколлегией

E-mail: buchstab@mendeleevo.ru, a.lazarev@bristol.ac.uk

15.01.2004 